\title{
Extended volume retinal vascular imaging with phase variance contrast optical coherence tomography
}

\author{
Jeff Fingler ${ }^{1, *}$, Daniel M. Schwartz ${ }^{2}$, and Scott E. Fraser ${ }^{1}$ \\ ${ }^{1}$ Dept. of Biology, California Institute of Technology, Pasadena, CA, \\ ${ }^{2}$ Dept. of Ophthalmology, University of California San Francisco, San Francisco, CA; \\ *Corresponding author: email: fingler@its.caltech.edu; phone: (626) 395-4499
}

\begin{abstract}
We present recent developments from a phase variance based motion contrast method of retinal vascular OCT imaging, called phase variance contrast optical coherence tomography (PV-OCT). Using a $25 \mathrm{kHz}$ spectral domain optical coherence tomography (SDOCT) system, the vascular visualization capabilities of this contrast method are demonstrated with composite images created from multiple data sets. Wide field vascular images extending over the fovea and optic nerve head are presented as well as microvascular retinal images over the fovea to demonstrate the trade-offs between imaging speed and vascular visualization.
\end{abstract}

Keywords: optical coherence tomography, phase contrast, microvasculature

\section{INTRODUCTION}

Non-invasive vascular imaging has the potential to be a valuable tool for diagnosis of retinovascular diseases, including but not limited to diabetic retinopathy and retinal vein occlusion. Non-invasive imaging can provide an alternative to conventional vascular imaging using fluorescein angiography, which is expensive, time-consuming and carries with it a risk of complications [1]. Optical coherence tomography (OCT) provides a non-invasive imaging technology which is ideally suited for usage as a screening instrument for retinal diseases and is capable of being adapted for vascular visualization. We have developed a vascular visualization technique called phase variance contrast optical coherence tomography (PV-OCT), which identifies regions of mobile scatterers based upon the variance of phase changes. This contrast method has previously been demonstrated in several different imaging scenarios: visualizing vasculature of the zebrafish [2], retinal vasculature of the mouse [3], and identifying microvasculature in small regions of the human retina [4]. This manuscript will focus on the visualization capabilities and limitations of PV-OCT and the applicability for vascular imaging over large retinal areas using a SDOCT system with an acquisition speed of $25 \mathrm{kHz}$, a speed comparable to current commercially-available retinal OCT systems.

There are numerous approaches in development for vascular visualization in the retina using OCT [5-10], but most of these methods are very similar to Doppler flow measurements, observing the axial component of scatterer flow within tissue between successive A-scans. This method has some fundamental limitations, making it difficult to observe slow flow or flow perpendicular to the imaging direction, especially microvascular features surrounding the fovea. As OCT acquisition speeds continue to increase, these types of flow measurements will see significant reductions in their ability to visualize slower axial flows. Phase variance contrast OCT (PV-OCT) produces vascular contrast based on the variance of phase changes between successive B-scans. The increased time separation between phase measurements in this method (as compared to the time between successive A-scans) allows for high sensitivity to visualize flows that are slow or transverse to the imaging direction, but requiring the sacrifice of quantitative flow information. Phase wrapping effects of vasculature regions with high axial flow can be beneficial in this contrast method, enhancing variance contrast through localized artifacts caused by high flow. Since the phase variance signal measured in PV-OCT is dependant on the time between B-scan measurements, this method has improved scalability to faster OCT acquisition rates compared to conventional Doppler OCT methods, which depend on the A-scan acquisition rate.

Ophthalmic Technologies XXI, edited by Fabrice Manns, Per G. Söderberg, Arthur Ho, Proc. of SPIE Vol. 7885, 78850I (C) 2011 SPIE · CCC code: $1605-7422 / 11 / \$ 18 \cdot$ doi: $10.1117 / 12.873893$ 


\section{EXPERIMENTAL SETUP AND RESULTS}

\subsection{PV-OCT Imaging System:}

The PV-OCT imaging system uses a spectral domain optical coherence tomography (SDOCT) design, operating at a line scan rate of $25 \mathrm{kHz}$, with an integration time of $36 \mu \mathrm{s}$. The system utilizes a superluminscent diode light source with a center wavelength of $840 \mathrm{~nm}$ and a spectral bandwidth of approximately $50 \mathrm{~nm}$. The retinal scanner is a modified scan head from a Zeiss Meditec STRATUS OCT3 imaging system, adjusted for a transverse spot size of approximately 15 20 microns.

PV-OCT imaging acquires the contrast signal through the usage of BM-scans (repeating B-scans over the same area for temporal information) and processing using procedures described previously [2-4]. The phase changes of the depthresolved scatterers between successive B-scans are used to calculate the phase variance contrast presented in this manuscript. Subject imaging was performed with undilated pupils and a basic head rest to minimize head motion. No form of bite bar or other head restraint was used for the acquired data sets, and no motion tracking was employed during image acquisition. PV-OCT image acquisition volumes were acquired in 4 seconds or less to reduce the effects of undesired eye motions.

\subsection{Scalability of PV-OCT for microvascular and vascular imaging}

The microvascular imaging capabilities of PV-OCT has previously been demonstrated in the human retina [4], but the retinal areas imaged were small $\left(0.5-1 \mathrm{~mm}^{2}\right)$ and have limited value for retinal vascular screening over a large field of view. Optimizing the vascular imaging capabilities in PV-OCT requires balancing the sampling density of the image to maximize the retinal scanning speed while ensuring that the retinal vasculature is still able to be visualized. Using a total acquisition time of 4 seconds, PV-OCT data sets were acquired over a large range of retinal scan areas, with each scan maintaining the same relative retinal sampling density in the $\mathrm{x}$ and $\mathrm{y}$ dimensions. Figure 1 demonstrates the microvascular visualization of the average phase variance contrast over the retinal depth anterior to the RPE, for retinal area sizes $1.25 \mathrm{~mm} \times 1.25 \mathrm{~mm}, 1.75 \mathrm{~mm}$ x $1.75 \mathrm{~mm}$ and $2 \mathrm{~mm} \times 2 \mathrm{~mm}$. In these cases, microvascular features are clearly distinguishable over the majority of these images. Additional processing and vascular isolation techniques can be used to improve the contrast of the vascularization relative to the rest of the retinal tissue.
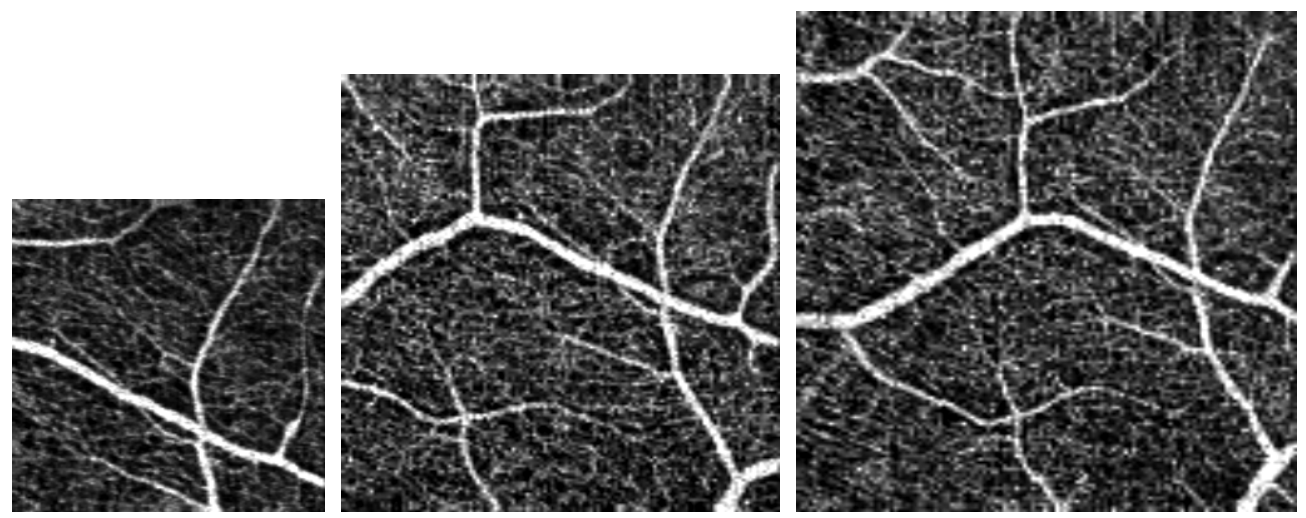

Figure 1. Microvascular visualization demonstrated for a range of retinal scan sizes. PV-OCT scans were acquired over retinal areas of $1.25 \mathrm{~mm} \times 1.25 \mathrm{~mm}$ (left), $1.75 \mathrm{~mm} \times 1.75 \mathrm{~mm}$ (middle) and $2 \mathrm{~mm} \times 2 \mathrm{~mm}$ (right).

The microvascular features are much harder to identify among larger scan areas. Figure 2 contains the average retinal phase variance contrast for retinal areas of $2.5 \mathrm{~mm} \times 2.5 \mathrm{~mm}$ and $3.5 \mathrm{~mm} \times 3.5 \mathrm{~mm}$. In these images the expected microvascular features are almost completely removed, resulting in the visualization of only the major retinal features. 
The vascularization visible within these images is consistent with the features observed by segmentation of the OCT intensity images.
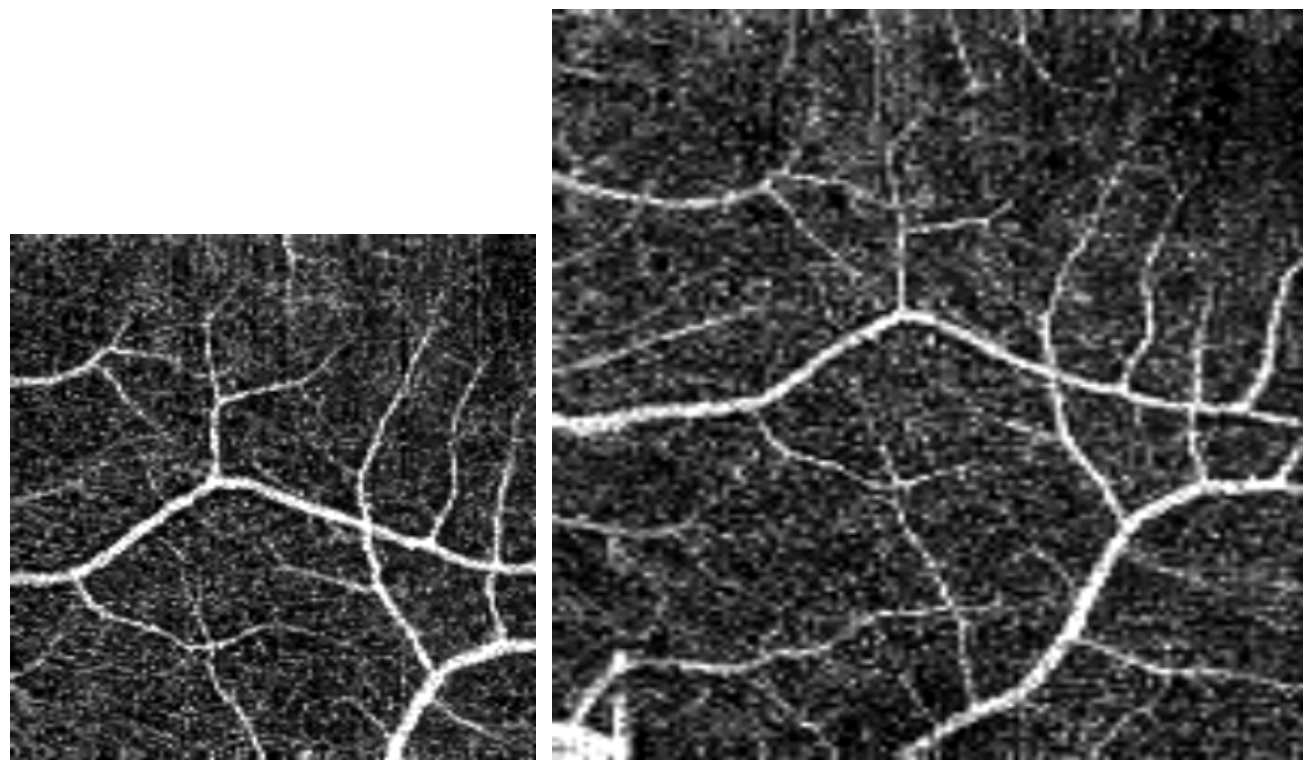

Figure 2. Vascular visualization demonstrated for larger retinal scan sizes. PV-OCT scans were acquired over retinal areas of $2.5 \mathrm{~mm} \times 2.5 \mathrm{~mm}$ (left) and $3.5 \mathrm{~mm} \times 3.5 \mathrm{~mm}$ (right).

Microvascular features can be visualized from the three-dimensional PV-OCT data though various forms of processing and presentation. The average phase variance contrast over the entire retinal depth presented in Figure 1 clearly shows the existence of microvasculature, but the image contrast is limited by the depth extent of the microvasculature contrast contribution. By segmenting the PV-OCT data over a smaller retinal depth, the average phase variance contrast can improve the microvascular contrast over a single retinal vascular layer. Figure 3 demonstrates the microvascular contrast improvement by isolating the anterior vascular layer in the retina and comparing it to the entire retinal average contrast presented in Figure 1.
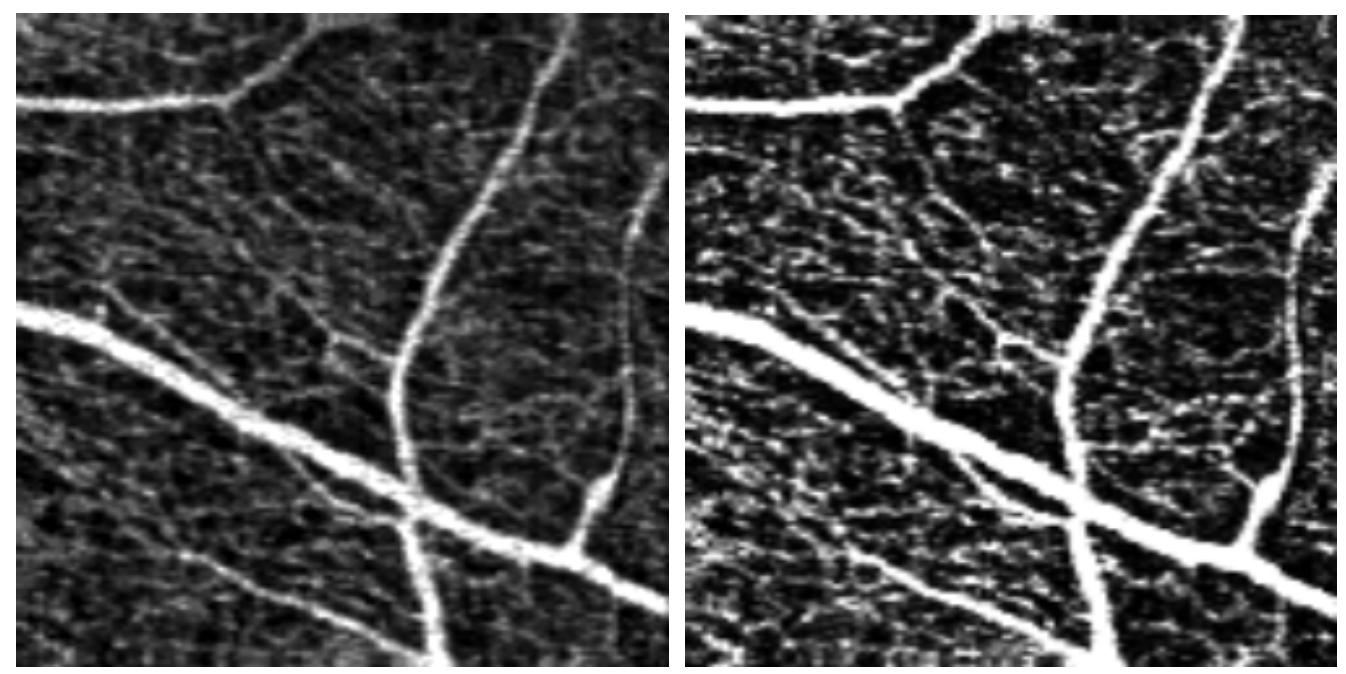

Figure 3. PV-OCT vascular visualizations of $1.25 \mathrm{~mm} \times 1.25 \mathrm{~mm}$ retinal scan area. Average variance contrast over the retina (left) demonstrates a different microvascular contrast to the average contrast over the anterior vascular layer in the retina (right). 


\subsection{Composite Imaging}

Limited by the acquisition speed of the OCT system, large scale microvascular images can be achieved with PV-OCT through composite imaging. By acquiring multiple data sets over overlapping regions of the retina, a composite image can be created from the individual data sets to extend the effective imaging area. Figure 4 demonstrates a composite vascular image created from 4 data sets, each of which was a PV-OCT scan over an area of $4 \mathrm{~mm} \times 4 \mathrm{~mm}$. Using a positional offset between images based upon mutual information of overlapping regions, the four individual images were combined to create a composite image of approximately $7 \mathrm{~mm} \times 7 \mathrm{~mm}$.
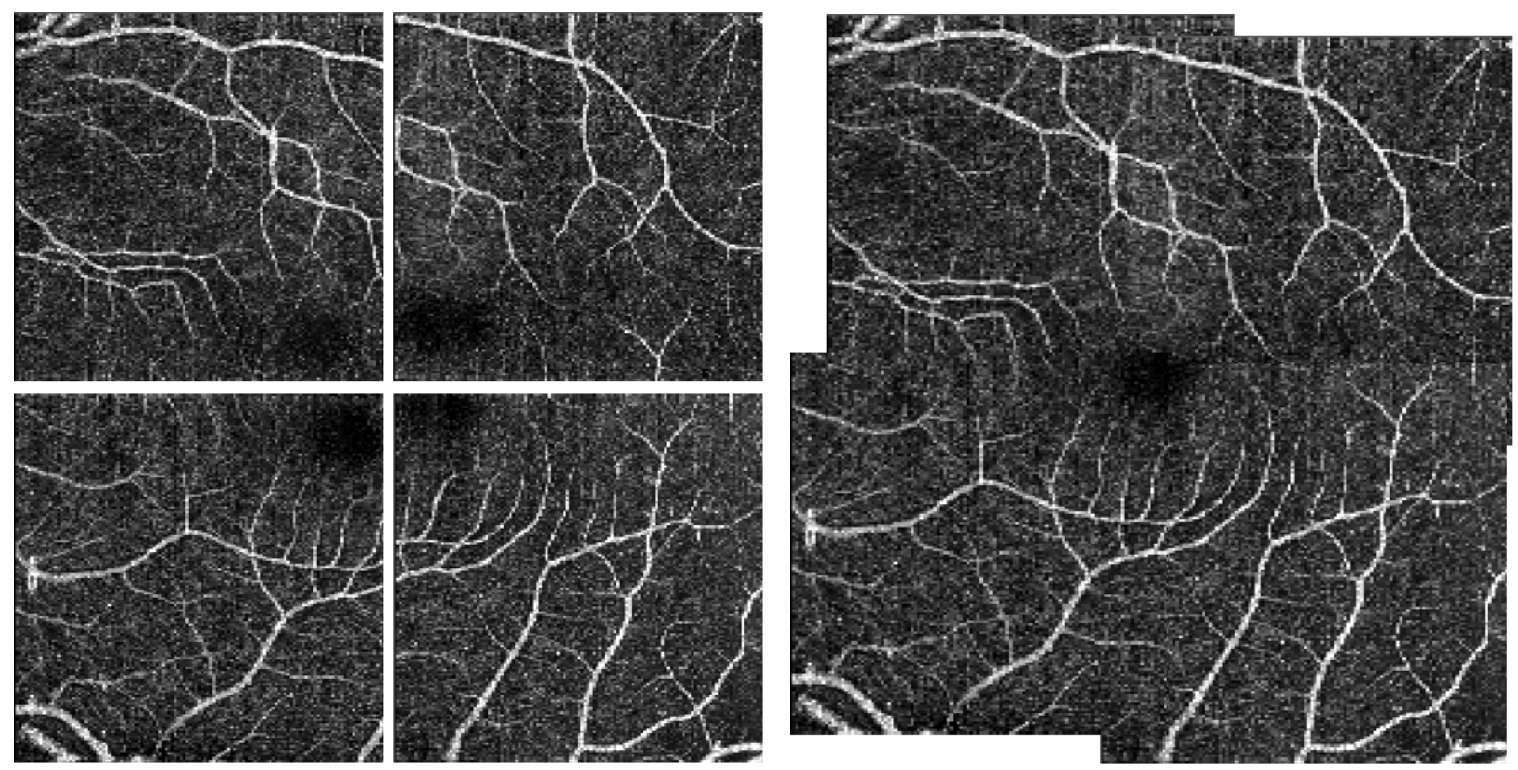

Figure 4. Multiple PV-OCT retinal vascular scans, each acquired over an area of $4 \mathrm{~mm} x 4 \mathrm{~mm}$. The individual scans (left) were combined to produce a composite image of approximately $7 \mathrm{~mm} \times 7 \mathrm{~mm}$ (right)

In this case, the composite image only required basic tiling due to the negligible amount of eye motion occurring during the PV-OCT scans. Microsaccadic eye movements and slower transverse eye motion can significantly affect the ability to create composite images, so these motions need to be minimized during acquisition or additional processing is required. With eye motion under control, large field of view composite images can be created with high sampling density and minimal distortion correction processing. Figure 5 demonstrates a retinal vascular composite image of approximately $10 \mathrm{~mm} \times 8 \mathrm{~mm}$, composed of overlapping PV-OCT data sets of $2 \mathrm{~mm} \times 2 \mathrm{~mm}$ retinal area, with each acquisition containing no microsaccades and minimal eye motion. As with the $2 \mathrm{~mm} \times 2 \mathrm{~mm}$ vascular scan presented in Figure 1, a lot of microvascular features are visible within the composite image but there are regions of limited contrast for visualizing the fine structure.

In practice, eye motion will be a much bigger issue that needs to be resolved for clinical composite imaging. Without the ability to track and compensate for fixation motion, composite imaging will be limited by the ability to identify and eliminate small fixation drifts within post-processing of the data. The presented composite alignment method uses large, relatively motion-free PV-OCT scans to align and composite smaller, microvascular PV-OCT images. Vascular landmarks based upon the branching and intersection of vascular features were identified within the large vascular scans and used to align the smaller microvascular scans. After an affine transform had been applied to bring the images into the same image space, a non-linear landmark warping using the Booksei method was performed. The resulting PV-OCT microvascular images were tiled to produce the composite image. Figure 6 demonstrates the results of the alignment method, compositing two $4 \mathrm{~mm} \times 4 \mathrm{~mm}$ PV-OCT vascular scans to produce the alignment framework, and using it to align and composite six PV-OCT microvascular scans of the anterior retinal vascular layer, each over an area of $1.75 \mathrm{~mm}$ $\mathrm{x} 1.75 \mathrm{~mm}$. 




Figure 5. Retinal vascular composite image of approximately $10 \mathrm{~mm} \times 8 \mathrm{~mm}$.
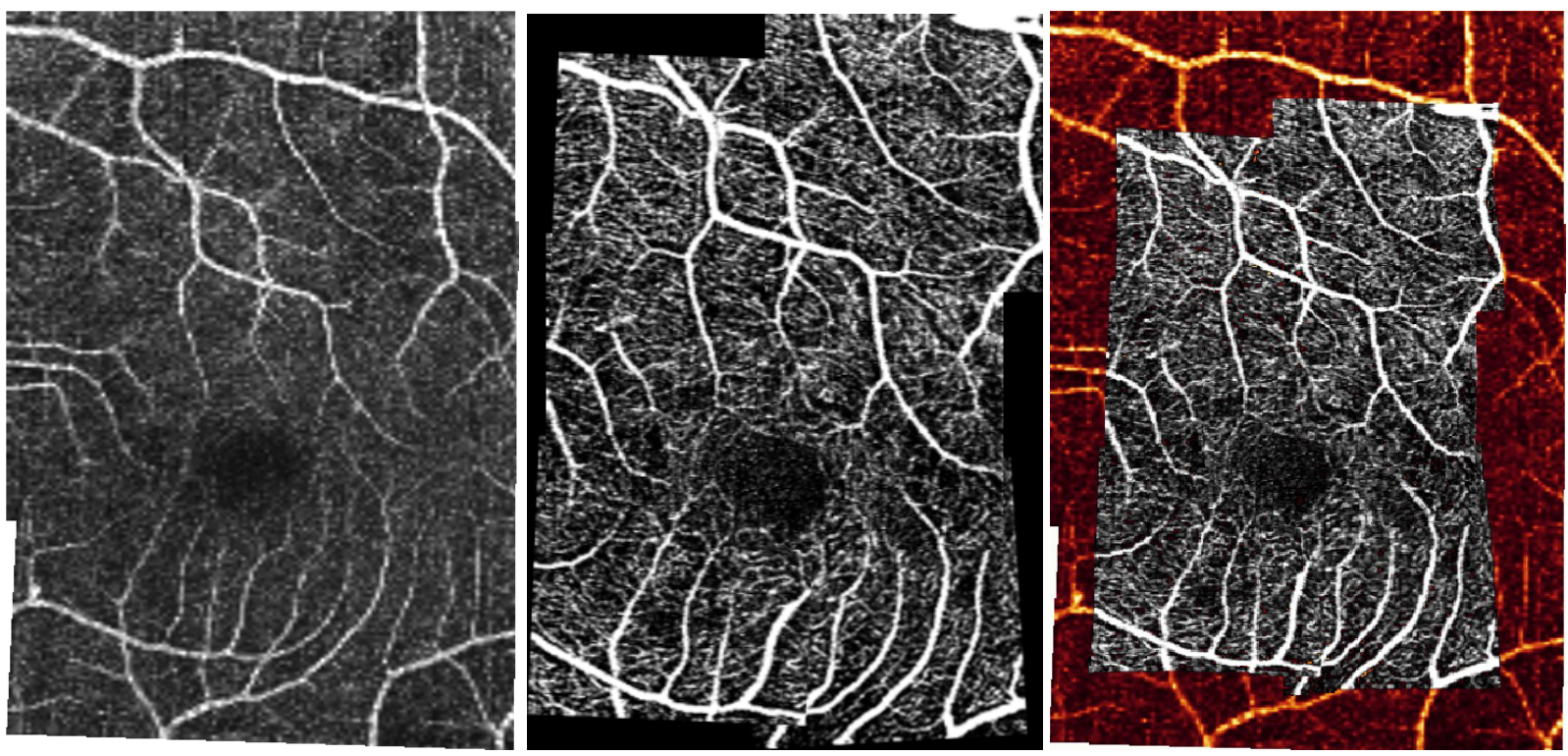

Figure 6. Creation of a microvascular PV-OCT composite image. Vascular alignment image created from two $4 \mathrm{~mm} \times 4 \mathrm{~mm}$ PV-OCT scans (left). Aligned microvascular composite image (middle). Overlay of the two images (right). 
The microvascular composite image presented in Figure 6 highlights many benefits, as well as some potential obstacles with this composite imaging method. While the image is almost distortion-free after alignment, there are several instances where the corrections do not fully compensate discrepancies at the boundaries of the individual data sets. This is caused by a limited number of vascular landmarks present within many regions of the individual microvascular PVOCT scans. Since vascular size and shape can be distorted through small eye motion, the vessels themselves are poor alignment markers, necessitating the use of vascular branching events as the landmarks. While vascular branching events serve as a much more robust set of landmarks, there are only a limited number of these events in many regions of the retina, especially around the fovea. Refinements of these alignment techniques, including the use of mutual information between neighbouring microvascular imaging regions, will continue to improve the robustness of this method.

The microvascular composite image is approximately $3 \mathrm{~mm}$ x $4.5 \mathrm{~mm}$, comprised of six PV-OCT scans of $1.75 \mathrm{~mm} \times$ $1.75 \mathrm{~mm}$, with overlapped imaged regions reducing the effective retinal scanning speed of the composite image. This redundant information is required to reduce the possibility of missing regions of the retina during imaging due to eye motion or an unintentional offset in the fixation position. Increased OCT acquisition rates would reduce the overlap within the composite image by allowing larger retinal scan areas, reducing the percentage of area devoted to overlapping data acquisition.

The major limiting factor to PV-OCT composite imaging, as well as many other retinal imaging techniques, is microsaccadic eye motion. Without active tracking and compensation, microsaccades cause large distortions and will likely cause regions of the retina to be missing within a single imaging scan. Even with proper registration and realignment, duplicate scans would be required to image the missing retinal sections, further reducing the effective imaging speed of the composite image. Further developments will offer many options for dealing with the effects of microsaccades and improving the robustness of PV-OCT composite imaging for use in clinical vascular imaging.

\section{CONCLUSION}

Phase variance contrast optical coherence tomography (PV-OCT) has demonstrated excellent microvascular imaging capabilities in the retina. Through composite imaging methods, a SDOCT system operating at $25 \mathrm{kHz}$ was able to produce large field of view retinal vascular images, limited only by the available field of view through the pupil. While PV-OCT could benefit from increased acquisition speeds without significant vascular contrast reduction, this experiment has demonstrated the potential of PV-OCT as an extended volume vascular imager while only operating at clinically available OCT acquisition speeds.

\section{ACKNOWLEDGEMENTS}

This research was supported by the Thome Foundation, Beckman Institute, and That Man May See Foundation.

\section{REFERENCES}

1. K. A. Kwiterovich, M. G. Maguire, R. P. Murphy, A. P. Schachat, N. M. Bressler, S. B. Bressler, and S. L. Fine, "Frequency of adverse systemic reactions after fluorescein angiography. Results of a prospective study," Ophthalmology 98(7), 1139-1142 (1991)

2. J. Fingler, D. Schwartz, C. Yang, and S. E. Fraser, "Mobility and transverse flow visualization using phase variance contrast with spectral domain optical coherence tomography," Opt. Express 15(20), 12636-12653 (2007)

3. J. Fingler, C. Readhead, D. M. Schwartz, and S. E. Fraser, "Phase-contrast OCT imaging of transverse flows in the mouse retina and choroid," Invest. Ophthalmol. Vis. Sci. 49(11), 5055-5059 (2008)

4. J. Fingler, R. J. Zawadzki, J. S. Werner, D. Schwartz, and S. E. Fraser, "Volumetric microvascular imaging of human retina using optical coherence tomography with a novel motion contrast technique," Opt. Express 17, 22190-22200 (2009) 
5. Y. Tao, K. Kennedy, and J. Izatt, "Velocity-resolved 3D retinal microvessel imaging using single-pass flow imaging spectral domain optical coherence tomography," Opt. Express 17(5), 4177-4188 (2009)

6. A. Szkulmowska, M. Szkulmowski, D. Szlag, A. Kowalczyk, and M. Wojtkowski, "Three-dimensional quantitative imaging of retinal and choroidal blood flow velocity using joint Spectral and Time domain Optical Coherence Tomography," Opt. Express 17(13), 10584-10598 (2009)

7. L. An, H. M. Subhush, D. J. Wilson, and R. K. Wang, "High-resolution wide-field imaging of retinal and choroidal blood perfusion with optical microangiography, " J. Biomed. Opt., Vol. 15, 026011 (2010)

8. L. Yu and Z. Chen, " Doppler variance imaging for three-dimensional retina and choroid angiography, " J. Biomed. Opt., Vol. 15, 016029 (2010)

9. T. Schmoll, C. Kolbitsch, and R. A. Leitgeb, "Ultra-high-speed volumetric tomography of human retinal blood flow," Opt. Express 17, 4166-4176 (2009)

10. S. Makita, T. Fabritius, and Y. Yasuno, "Quantitative retinal-blood flow measurement with three-dimensional vessel geometry determination using ultrahigh-resolution Doppler optical coherence angiography," Opt. Lett. 33, 836$838(2008)$ 